\title{
Review of: "Real-time geospatial analysis identifies gaps in COVID-19 vaccination in a minority population"
}

\author{
Gregory Umeh ${ }^{1}$ \\ 1 World Health Organization WHO
}

Potential competing interests: The author(s) declared that no potential competing interests exist.

Thank you for asking me to review the manuscript.

The manuscript is relevant, and timely, as the global public health community ramp up COVID-19 vaccination, and other preventive measures.

Title:

The title could do with setting (place and time).

Introduction:

Too short, not thorough, and nothing on COVID-19 epidemiology (global and local), and relevance of nonpharmaceutical interventions (handwashing, mask etc.), even with high vaccination coverage.

\section{Methods:}

Sound methods and clear procedures

\section{Results:}

It will de desirable to know the education and income of the vaccinees or study population

\section{Discussion:}

The authors opened the discussions with repetition of the methods. It should not be so!

The authors should make sense of their results vis-a vis the hypotheses. Were the hypotheses met and why? And if not not met, why? The relationship of their results to other studies should be discussed, as well as the implications/relevance of the study. The authors should state the limitations of the study and ends with recommendations and conclusion.

\section{General Comment:}

The language is clear and proper. The work could do with line numbering to make review easy. 